\title{
Study on Surge and Rotating Stall in Axial Compressors* (A Numerical Model and Parametric Study for Multiblade-Row Compressors)
}

\author{
Hiroshi ISHII** and Yasushige KASHIWABARA***
}

\begin{abstract}
This study investigates the effects of various parameters on the inception and poststall characteristics of surge and rotating stall in multistage axial compressors, using a numerical model for multiblade-row compressors. The parameters described in this study are the shape of the total pressure loss and deviation angle characteristics, IGV (inlet guide vane) outlet flow angle, inlet flow distortion, and bleeding. It is found that there are two types of rotating stall differing in magnitude, that inlet distortion and the deterioration in compressor performance reduce the inception margin of unstable behavior, and that there is an optimal bleeding that can optimize the inception margin of unstable behavior. The usefulness of the present model is examined through numerical investigations, and the validity of the model is confirmed by comparing predicted results obtained using the model with test results obtained using a singlestage compressor and a 3 -stage compressor.
\end{abstract}

Key Words: Axial Compressor, Surge, Rotating Stall, Semiactuator Disc Theory, Numerical Simulation, Parametric Study

\section{Introduction}

Predicting unstable behaviors such as surge and rotating stall is very important when designing the multistage axial compressors used for gas turbines. These behaviors cause not only poor compressor performance, but also excessive vibration of the blades and shaft, possibly leading to forced stoppage of the gas turbine or to blade failure. In predicting these behaviors, emphasis is placed on estimating the inception points and on quantitatively evaluating the dynamic flow velocity and pressure inside the compressor once these behaviors occur. Estimating the inception points is important in evaluating surge and

* Received 1st March, 1995. Japanese originals: Trans. Jpn. Soc. Mech. Eng., Vol. 59, No.560, B (1993), pp. 1127-1133 ( Received 24th September, 1992); Vol. 59, No. 568, B (1993), pp. 38381845 (Received 26th March, 1993)

** Mechanical Engineering Research Laboratory, Hitachi, Ltd., 502 Kandatsu, Tsuchiura, Ibaraki 300, Japan

*** Kanagawa Institute of Technology, 1030 Shimoogino, Atsugi, Kanagawa 243-02, Japan rotating stall margins of unsteady performance. Evaluating the dynamic flow velocity and pressure is essential in estimating blade vibration during rotating stall, especially during low-speed operation.

Several features of surge and rotating stall in multistage axial compressors presented by Hagiwara et al. ${ }^{(1)}$ and Matsuura et al. ${ }^{(2)}$ indicate the possibility of these behaviors occurring simultaneously. However, in most research on unstable behaviors of axial compressors, surge and rotating stall have been considered separately $y^{(3),(4)}$. A theoretical model representing a combination of surge and rotating stall in axial compressors was presented by Greitzer and Moore ${ }^{(5)}$ and Moore ${ }^{(6)}$ and using simulation as well as the relationship of the patterns of unstable behaviors to compressor parameters shows that there are several patterns of simultaneous occurrence. Another theoretical model presented by Ishii and Kashiwabara ${ }^{(7)}$ extends the previous model to include the simultaneous effects of inlet distortion and valve movement on the stall transient behavior of axial compressors. Ishii and Kashiwabara ${ }^{(8)}$ proposed a numerical model that expands the previous theoretical model by expressing circumferential flow velocity distortion for rotating 
stall in the form of a high-order Fourier series and taking into account the nonlinearity of equations for the angular variable. Although these models help us understand the basic mechanism of surge and rotating stall, they are not precise enough for practical application to the design of multistage compressors. One of the main reasons for this is that the effect of compres. sibility is concentrated in a plenum, which is valid for single-stage compressors and to a certain degree, compressors with a small number of stages. Another reason is that only pressure-rise characteristics are considered in the blade row, whereas two characteristics are actually needed.

The purpose of the work is to develop a method of predicting both surge and rotating stall and that is applicable to the design of multistage axial compres sors. This paper proposes a numerical model that expands on the previous fundamental model in order to represent surge and rotating stall in multistage axial compressors more accurately. In this model compressibility is distributed throughout the compressor, and the blade rows are replaced by semiactuator disks of finite width. This paper uses the model to investigate the effects of various parameters on the inception and post-stall characteristics of surge and rotating stall in multistage axial compressors. The parameters include the shape of the total pressure loss and deviation angle characteristics, IGV outlet flow angle, inlet flow distortion, and bleeding. This paper also examines the validity of the model by comparing its predicted results using with test results for a singlestage compressor and a three-stage compressor.

\section{Nomenclature}

$A$ : compressor duct area

$a, b:$ Fourier coefficient of disturbance

$a_{s}:$ speed of sound

$B$ : B parameter

$C$ : length of blade cord

$c^{\prime}$ : length of throttle

$H$ : semiheight of cubic characteristic

$H_{T}$ : total enthalpy

$K_{T}$ : throttle coefficient

$L_{T}$ : exit duct length

$M$ : margin of unstable behavior

$P$ : static pressure

$P_{T}:$ total pressure

$Q_{B}$ : amount of bleeding

$R$ : gas constant

$r$ : mean wheel radius

$T:$ static temperature

$t$ : time for wheel to rotate one radian

$U$ : wheel speed at mean diameter

$u$ : axial flow velocity
$V:$ total flow velocity

$v$ : circumferential flow velocity

$W$ : semiwidth of cubic characteristic

$x$ : axial coordinate measured in compressor mean radii

$\alpha$ : flow angle

$\beta$ : incidence/deviation angle

$\gamma$ : stagger angle/blade angle

$\varepsilon$ : axial velocity of inlet distortion

$\zeta$ : total pressure loss coefficient

$\theta$ : angular coordinate

$\kappa$ : ratio of specific heats

$\xi$ : deviation angle

$\pi$ : pressure ratio

$\rho$ : density of fluid

Subscripts

$d$ : dimensional variable/design point

$i$ : node number

$d$ : nondimensional base variable

$O$ : nondimensional standard variable

$R$ : variable in rotary coordinate

$T$ : total condition

- : annulus ${ }^{-}$averaged variable

$\sim$ : annulus -disturbance variable

\section{Numerical Method}

\section{1 Numerical model}

In the fundamental model ${ }^{(7)}$, the effect of compressibility is concentrated in a plenum acting as an adiabatic air spring, and the fluid inertia is considered to be the total length of the flow path. This is the onedegree- of-freedom vibration system. The model also uses the steady pressure-rise characteristics for all the compressor stages as the compressor characteristics applied to a semiactuator disc. This assumption is valid for single-stage compressors and compressors with a small number of stages. The extended model, used to obtain a more accurate representation of both surge and rotating stall in multistage axial compressors, is a so-called multi-degree- of-freedom vibration system, where the compressibility is distributed throughout the compressor, and the characteristics of total pressure loss and deviation angle are used for each blade row.

In the extended model, a compressor is composed of three elements : ducts, blade rows and a throttle, as shown in Fig. 1. The duct element comprises an inlet duct, ducts between the blade rows, and an exit duct. For the duct elements, the model takes two-dimensional inviscid compressible flow in the axial and circumferential directions into account. The flow field is represented using a full set of fluid equations consisting of a continuity equation, equations of motion without viscosity, an energy equation, and an equation 


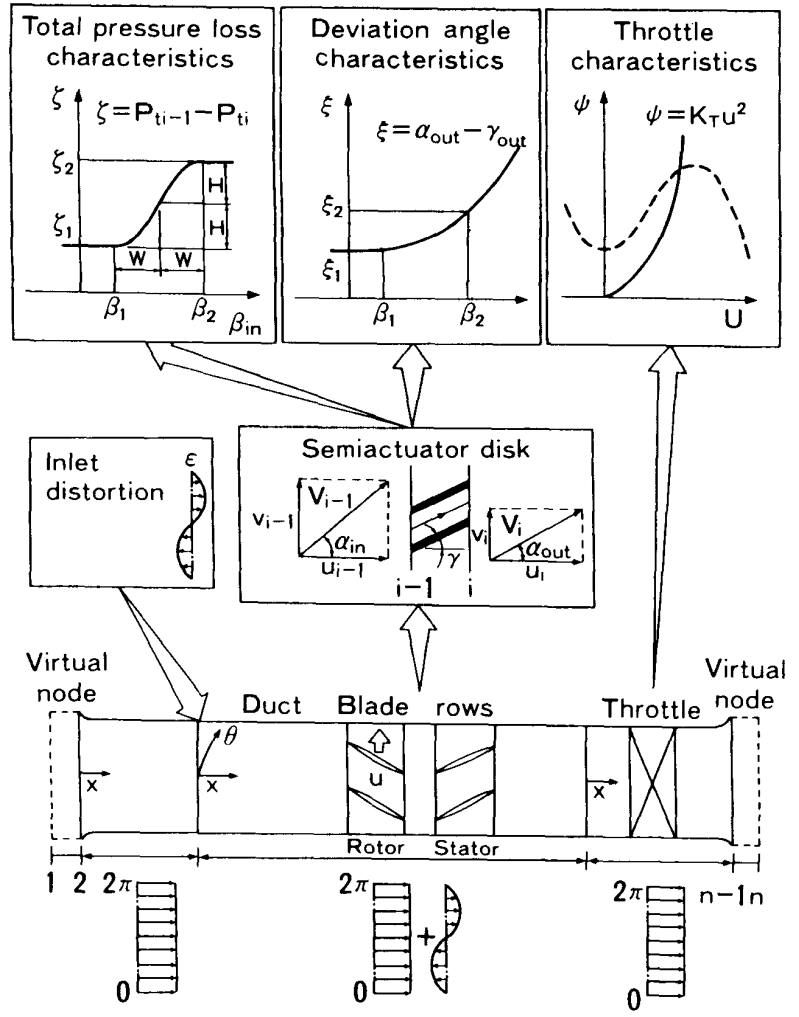

Fig. 1 Outline of model

of state. The blade row elements comprises rotor blade rows, stator blade rows, IGV row, and exit guide vane (EGV) rows. The blade rows are replaced by semiactuator disks; that is, by cascades of flat plates, where the blade pitch is infinitely small and the blade cord length is finite. The blade row characteristics are expressed in forms of total pressure loss and deviation angle as functions of incidence angle in the blade stagger direction. For the blade row elements, the relations for total pressure loss and deviation angle are used instead of the equations of motion for the duct elements. For the throttle element, where the flow is assumed to be one-dimensional and incompressible, the model uses the compressible total pressure drop instead of the equations of motion.

As shown in Fig. 1, variables such as flow velocity and pressure are divided into a circumferentially averaged component, which describes flow oscillation due to surge, and a circumferentially distorted component, which describes circumferential flow distortion due to rotating stall.

\section{2 Formulation of equations for numerical model}

Since the present model takes compressible flow into account, the flow field inside the compressor can be represented using the following set of fluid equations.

Continuity equation :

$$
\frac{\partial \rho}{\partial t}+\frac{1}{A} \frac{\partial}{\partial x}(A \rho u)+\frac{1}{r} \frac{\partial}{\partial \theta}(\rho v)=0
$$

Equations of motion:

$$
\begin{aligned}
& \frac{\partial u}{\partial t}+u \frac{\partial u}{\partial x}+v \frac{1}{r} \frac{\partial u}{\partial \theta}=-\frac{1}{\rho} \frac{\partial P}{\partial x} \\
& \frac{\partial v}{\partial t}+u \frac{\partial v}{\partial x}+v \frac{1}{r} \frac{\partial v}{\partial \theta}=-\frac{1}{\rho} \frac{1}{r} \frac{\partial P}{\partial \theta}
\end{aligned}
$$

Energy equation :

$$
\frac{\partial H_{T}}{\partial t}+u \frac{\partial H_{T}}{\partial x}+v \frac{1}{r} \frac{\partial H_{T}}{\partial \theta}=\frac{1}{\rho} \frac{\partial P}{\partial t}
$$

where

$$
H_{T}=i+\frac{V^{2}}{2}=\frac{k}{k-1} R T+\frac{u^{2}}{2}+\frac{v^{2}}{2}
$$

Equation of state of perfect gas :

$$
P=\rho R T
$$

All the variables are nondimensionalized as follows.

$$
\begin{aligned}
& u^{\prime}=\frac{u}{U_{0}}, v^{\prime}=\frac{v}{U_{0}}, t^{\prime}=\frac{U_{0}}{r_{0}} t, x^{\prime}=\frac{x}{r_{0}}, p^{\prime}=\frac{P}{\rho_{0} U_{0}^{2}}, \\
& r^{\prime}=\frac{r}{r_{0}}, A^{\prime}=\frac{A}{A_{0}}, R^{\prime}=\frac{R T_{0}}{U_{0}^{2}}, T^{\prime}=\frac{T}{T_{0}}
\end{aligned}
$$

Substituting Eq. ( 5 ) and the equation obtained by differentiating Eq. ( 6 ) with respect to time, $t$, into Eq. (4), the following equation is obtained.

$$
\begin{aligned}
& \frac{1}{k-1} R \frac{\partial T}{\partial t}+u \frac{\partial u}{\partial t}+v \frac{\partial v}{\partial t}-\frac{R T}{\rho} \frac{\partial \rho}{\partial t} \\
& =\frac{k}{k-1} R u \frac{\partial T}{\partial x}-u^{2} \frac{\partial u}{\partial x}-u v \frac{\partial v}{\partial x} \\
& -\frac{1}{r} \frac{k}{k-1} R v \frac{\partial T}{\partial \theta}-\frac{1}{r} u v \frac{\partial u}{\partial \theta}-\frac{1}{r v} v^{2} \frac{\partial v}{\partial \theta}
\end{aligned}
$$

The equations for the blade row, which can be divided into several sections, are obtained using the total pressure loss in the blade stagger direction between the inlet and outlet, and by taking the effect of the inertia of the fluid into account :

$$
\begin{aligned}
& P_{T_{i-1}}-P_{T_{i}}-c \frac{\rho}{\cos \gamma} \frac{\partial u}{\partial t}=\zeta\left(\alpha_{\mathrm{in}}-\gamma_{\mathrm{in}}\right) \times \frac{1}{2} \rho_{\mathrm{ln}} V_{\mathrm{in}}^{2} \\
& P_{T}=P\left(1+\frac{\kappa-1}{2 \kappa} \frac{\rho}{p} V^{2}\right)^{k /(k-1)}
\end{aligned}
$$

where $\zeta$ denotes the total pressure loss coefficient as a function of incidence angle $\beta$, as shown in Fig. 2 . It is possible to change the total pressure loss distribution according to the axial position in the blade channel by fitting to the actual distribution.

The other equation for the blade row is the following relation between the deviation angle and the incidence angle:

$$
\beta_{\text {out }}=\alpha_{\text {out }}-\gamma_{\text {out }}=\xi\left(\alpha_{\text {in }}-\gamma_{\text {In }}\right), \frac{v_{\text {out }}}{\mathcal{u}_{\text {out }}}=\tan \beta_{\text {out }}
$$

where $\xi$ denotes the deviation angle as a function of the incidence angle.

The present method neglects the dynamic characteristics of the total pressure loss coefficient $\zeta$ and the deviation angle function $\xi$, which are caused mainly by a time delay due to the behavior of the boundary layer on the blade surface. The investigation of these 
dynamic effects is left for future study. For the blade row, Eqs. (9), (10) and (11) are used instead of the equations of motion (2) and ( 3 ).

Because Eqs. ( 2 ) - (11) are valid for relative coordinates, the following coordinate conversion between stationary and rotary coordinates is necessary for the rotor blade row.

$$
\bar{v}_{R}=\bar{v}-1, \frac{\partial u}{\partial t}=\frac{\partial u_{R}}{\partial t}-\frac{\partial u}{\partial \theta}, \frac{\partial v}{\partial t}=\frac{\partial v_{R}}{\partial t}-\frac{\partial v}{\partial \theta}
$$

For the throttle, where the flow direction must be axial, we can assume the parabolic function characteristics shown in Fig. 1, from which we obtain the following equation for the total pressure drop.

$$
P_{T i-1}-T_{T i}-c^{\prime} \rho \frac{\partial u}{\partial t}=\frac{1}{2} K_{T} \rho_{i-1} u_{i-1}^{2}
$$

For the throttle, this equation is used instead of Eq. (2).

The boundary conditions upstream and downstream of the compressor are assumed as follows.

$$
\frac{\partial \bar{u}}{\partial x}=\frac{\partial T}{\partial x}=0, \frac{\partial P_{T}}{\partial t}=0
$$

The following boundary conditions are also assumed for a point, (the inlet of the exit plenum, for example) behind the last blade row.

$$
\bar{v}=0, \tilde{u}=\tilde{v}=\tilde{p}=\tilde{\rho}=0
$$

The circumferential inlet distortion $\varepsilon$, which appears in front of the first blade row, is given by the following boundary conditions.

$$
\tilde{u}=\varepsilon, \frac{\partial \tilde{u}}{\partial x}=0
$$

\section{3 Numerical method for solution}

All the variables are functions of time $t$, axial coordinate $x$, and angle coordinate $\theta$. To obtain values for them, the finite difference method is used for the derivatives of time and the axial coordinate, and the Galerkin procedure is used for the angular variable. The variables $u, v, P, \rho$, and $T$ can be divided into circumferentially averaged components, which describe flow oscillation due to surge, and circumferentially distorted components, which describe circumferential flow distortion due to rotating stall. The circumferential distortions of the variables should be periodic functions of $\theta$ and their average for the circumferential direction should be zero. Thus the solutions of the governing equations can be expressed as a Fourier series. For example, the axial flow velocity can be expressed in the form.

$$
\begin{aligned}
& u(t, x, \theta)=\bar{u}(t, x)+\bar{u}(t, x, \theta) \\
& \quad=\bar{u}(t, x)+\sum_{k=1}^{n}\left\{a_{u k}(t, x) \cos k \theta\right. \\
& \left.+b_{u k}(t, x) \sin k \theta\right\}
\end{aligned}
$$

Because the purpose of this study is to examine the basic function of the proposed model, only the first order of the Fourier series is considered. Moreover, the circumferential disturbance of temperature $T$ is assumed to be negligible in the case of rotating stall.

$$
T(t, x, \theta)=\bar{T}(t, x)
$$

Substituting the solutions into the governing equations to obtain a residual of constant, $\sin \theta$ and $\cos \theta$ using the Galerkin procedure, equations without derivatives of the circumferential coordinate are obtained. For example, Eq. ( 2 ) can be written as follows.

$$
\begin{aligned}
& \frac{\partial \bar{u}}{\partial t}+\bar{u} \frac{\partial \bar{u}}{\partial x}+\frac{1}{2}\left(a_{u} \frac{\partial a_{u}}{\partial x}+b_{u} \frac{\partial b_{u}}{\partial x}\right) \\
& +\frac{1}{2}\left(a_{v} b_{u}-b_{v} a_{u}\right)=-\frac{1}{\bar{\rho}} \frac{\partial \bar{p}}{\partial x} \\
& +\frac{1}{\rho^{2}}\left(\frac{1}{2} a_{\rho} \frac{\partial a_{\rho}}{\partial x}+\frac{1}{2} b_{\rho} \frac{\partial b_{\rho}}{\partial x}\right) \\
& \frac{\partial b_{u}}{\partial t}+\left(\bar{u} \frac{\partial b_{u}}{\partial x}+b_{u} \frac{\partial \bar{u}}{\partial x}\right)-\frac{1}{r} \bar{u} a_{u} \\
& =-\frac{1}{\bar{\rho}} \frac{\partial b_{P}}{\partial x}+\frac{1}{\rho^{2}} b_{\rho} \frac{\partial \bar{p}}{\partial x} \\
& \frac{\partial a_{u}}{\partial t}+\left(\bar{u}-\frac{\partial a_{u}}{\partial x}+a_{u} \frac{\partial \bar{u}}{\partial x}\right)+\frac{1}{r} \bar{u} b_{u} \\
& =-\frac{1}{\bar{\rho}} \frac{\partial a_{P}}{\partial x}+\frac{1}{\rho^{2}} a_{\rho} \frac{\partial \bar{p}}{\partial x}
\end{aligned}
$$

The equations obtained include only derivatives of the axial coordinate and time. The derivatives of the axial coordinate are obtained by applying the finite difference method to variables at discrete nodes in the axial direction. To obtain a stable calculation, the upwind difference is used for the variable the time derivative of which is included in the equations, whereas the central difference is used for the other variables. For example, Eq. (19) can be transformed into the following equation.

$$
\frac{\partial \bar{u}_{i}}{\partial t}+\bar{u}_{i} \frac{\bar{u}_{i}-\bar{u}_{i-1}}{\Delta x}+\cdots=-\frac{1}{\bar{\rho}_{i}} \frac{\bar{p}_{i+1}-\bar{p}_{i-1}}{2 \Delta x}
$$

For node $i$ with bleeding $Q_{B}$, the following continuity equation for steady flow is used.

$$
\begin{aligned}
& \frac{\partial \bar{\rho}_{i}}{\partial t}+\frac{1}{A_{i}} \frac{\bar{\rho}_{i+1}}{}\left(A_{i+1} \frac{\left.\bar{u}_{i+1}+Q_{B}\right)-\bar{\rho}_{i-1} A_{i-1} \bar{u}_{i-1}}{\Delta x}\right. \\
& \quad+\frac{1}{r_{i}} \frac{\partial}{\partial \theta}\left(\rho_{i} v_{i}\right)=0
\end{aligned}
$$

Because the equations, especially that for total pressure loss, are strongly nonlinear for the axial coordinate, the following smoothing method (for the variable $u$, for example) may be used to maintain stability :

$$
u_{i}^{\prime}=\frac{k u_{i}+u_{i-1}+u_{i+1}}{k+2}
$$

where $k$ is a smoothing coefficient selected because of its strength of nonlinearity. In this study, the value of $k$ is determined by checking the stability in various cases, to be 100 for the blade rows and 1000 for the ducts.

To express the boundary conditions upstream and downstream of the compressor, virtual nodes outside the compressor are considered as shown in Fig. 1. 
Then the boundary conditions are written as follows.

$$
\begin{aligned}
& u_{1}=u_{2}, T_{1}=T_{2}, P_{T 1}=\text { const } \\
& u_{n}=u_{n-1}, T_{n}=T_{n-1}, P_{T n}=\mathrm{const}
\end{aligned}
$$

Regarding the derivative of time, the conventional forward difference is used as follows.

$$
u^{m+1}=u^{m}+\frac{\partial u}{\partial t} \Delta t
$$

To obtain a stable solution using the finite difference method, it is important to choose an appropriate time step $\Delta t$ for the axial mesh size $\Delta x$. The following condition should be satisfied.

$$
\Delta t \leqq \frac{\Delta x}{\sqrt{K \bar{R}} \overline{\bar{T}}+\overline{\bar{u}}}
$$

\section{Calculation Conditions}

The calculation conditions are shown in Figs. 2 4. Figure 2 shows an overall view of the compressor and a magnified view of a blade row section with a representative blade profile at mean wheel radius for each blade row. The compressor consists of an entrance duct, blade rows, a throttle duct, and a throttle. The blade rows begin with an IGV and end with an EGV. The number of stages between 1 and 3 is selected. The design speed is $13,215 \mathrm{rpm}$ and the pressure ratio is assumed 1.3 for each stage. As shown in Fig. 2, for the $3^{-}$stage compressor, three different speeds are selected. Three cases are considered for the IGV outlet blade angle in order to investigate the effect of change in mass flow on the charac-

\begin{tabular}{|c|c|c|c|}
\hline Case No. & 1 & 2 & 3 \\
\hline$\gamma_{\mathrm{IGV} \text { (deg) }}$ & 10.0 & 17.0 & 24.0 \\
\hline
\end{tabular}
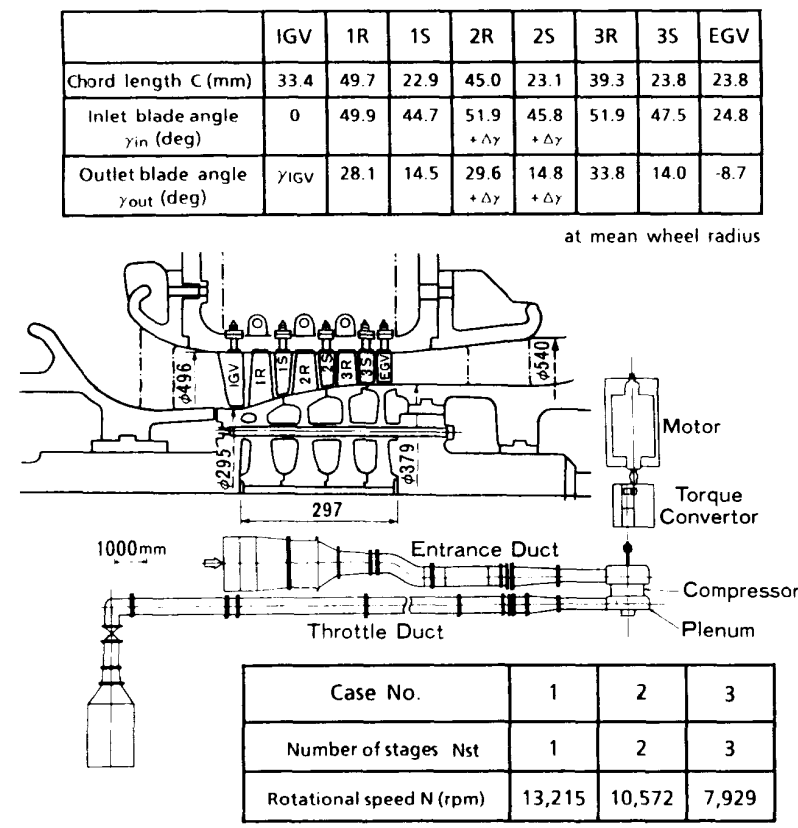

Fig. 2 Configuration of compressor used for calculation teristics of rotating stall. Two cases are assumed for the blade angle of the second stage when a 2 -stage compressor is selected. This condition is used to investigate the bleeding effect on the characteristics of rotating stall by causing a choke state in the second stage, which is often observed in multistage compressors. Figure 3 shows the characteristics for three cases of total pressure loss $\zeta$ and deviation angle $\xi$ against incidence angle $\beta_{\mathrm{in}}$. The values of $\zeta$ and $\xi$ in an unstalled condition are determined by referring to the test results for the NACA $65^{-}$series profile. The values of $\zeta$ and $\xi$ are assumed to be constant for $\beta_{\text {in }}$ $\leqq 8$ degrees. Because the characteristics in a stalled condition are unknown, a cubic function is assumed for the total pressure loss, and a parabolic function is assumed for the deviation angle. As shown in Fig. 3, the three cases differ with respect to the value of $\beta_{2}$. The value of $\zeta$ when $\beta_{\text {in }} \geqq \beta_{2}$ is assumed to be 0.8 . The shapes of these characteristics are related to the compressor stall characteristics. As $\beta_{2}$ decreases, the stalling tend to occur. This corresponds to a deterioration in compressor performance due to increasing blade tip gap and fouling on the blade surface. Each blade row is divided, in the blade stagger direction, into 8 elements for a rotor blade and 4 elements for a stator blade. The distribution of total pressure loss is assumed to be the same as that in Fig. 3, where the total pressure loss near the leading edge is estimated to be relatively large by considering the stall conditions. Figure 4 shows the calculation conditions for inlet flow velocity distortion, bleeding, throttle duct length, and throttle coefficients. The inlet circumfer-

\begin{tabular}{|c|c|c|c|}
\hline Case No. & 1 & 2 & 3 \\
\hline$\beta_{2}$ (deg) & 30.0 & 37.5 & 45.0 \\
\hline
\end{tabular}

\begin{tabular}{|c|c|c|}
\hline Blade & Rotor & Stator \\
\hline $\mathrm{n}_{\mathrm{B}}$ & 8 & 4 \\
\hline
\end{tabular}
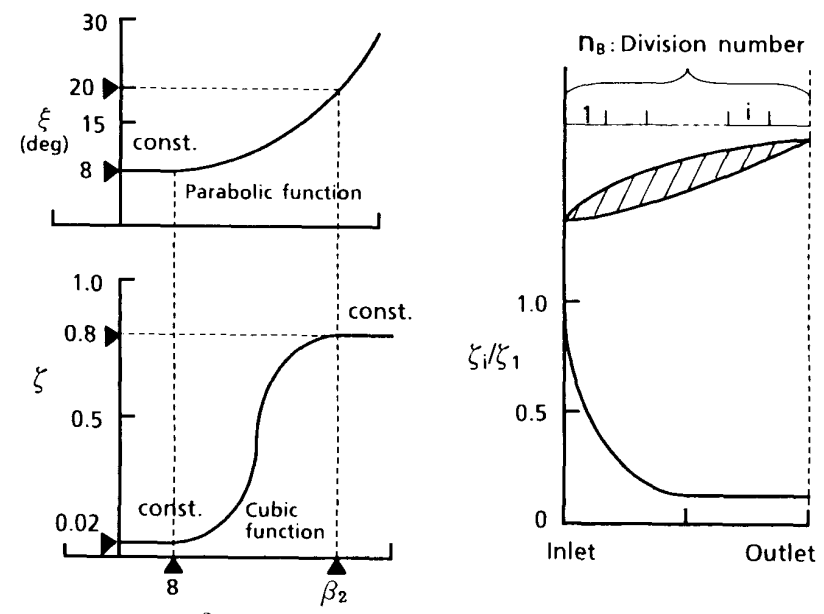

$\beta_{\text {in }}$ (deg)

Fig. 3 Calculation conditions for total pressure loss and deviation angle characteristics 


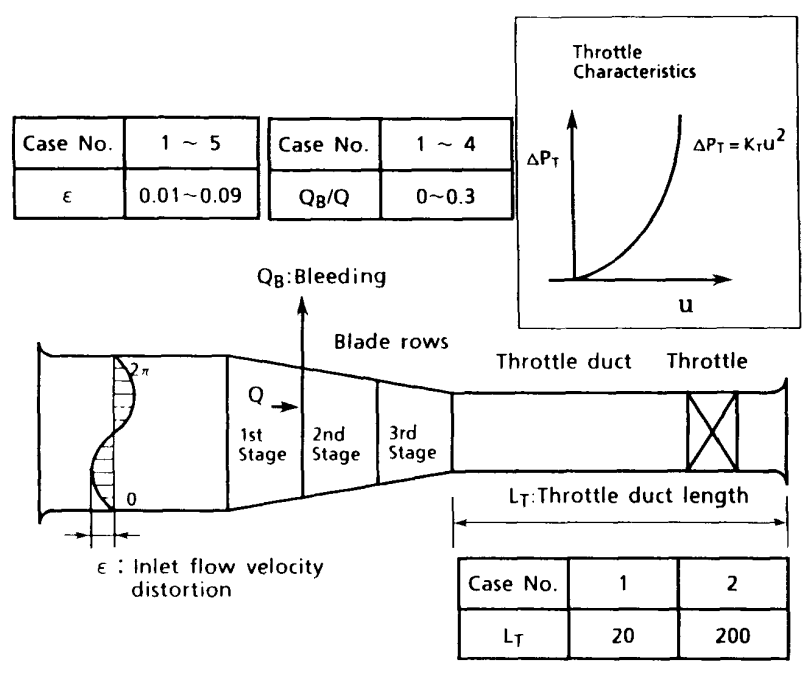

Fig. 4 Calculation conditions for inlet distortion, bleeding, throttle duct length, and throttle coefficient

ential distortion of the axial flow velocity in front of IGV is fixed, and the amplitude of the distortion is changed in order to investigate the inception points of unstable behaviors. Bleeding between the first and second stages, is changed from 0 to $30 \%$ of the main flow. Two lengths of the throttle duct are considered in order to investigate the modes of post-stall unstable behaviors.

The throttle coefficient $K_{T}$ is varied in order to investigate the steady and unsteady compressor performance and inception points. In this study, the following values are used for $\Delta t$ and $\Delta x$.

$$
\Delta t=0.002 \sim 0.005 ; \Delta x=0.02 \sim 1.00
$$

\section{Calculation Results}

\section{1 Throttle duct length effect}

Figure 5 shows steady and unsteady compressor characteristics, which are represented by plotting the pressure ratio $\pi$ against the axial flow velocity $u$ for a single-stage compressor composed of an IGV, rotor and stator blade rows, and an EGV. These characteristics were obtained by changing the throttle coefficient. Results for two lengths of the throttle duct are compared. One length is $L_{T}=20$, corresponding to a small plenum (i.e., a small B-parameter in the fundamental model) and the other is $L_{T}=200$, which corresponds to a large plenum. No unstable behavior occurs on the right side of point $S$, and for both cases, instability occurs at point $S$. There is little difference between the inception points for surge and for rotat. ing stall. When $L_{T}=200$, the post ${ }^{-}$stall trajectory become a large loop, and the time history of local flow velocity at the first rotor blade, which is shown in the upper part of the figure, is a saw-wave. In this case, surge occurs. When $L_{T}=20$, rotating stall occurs on

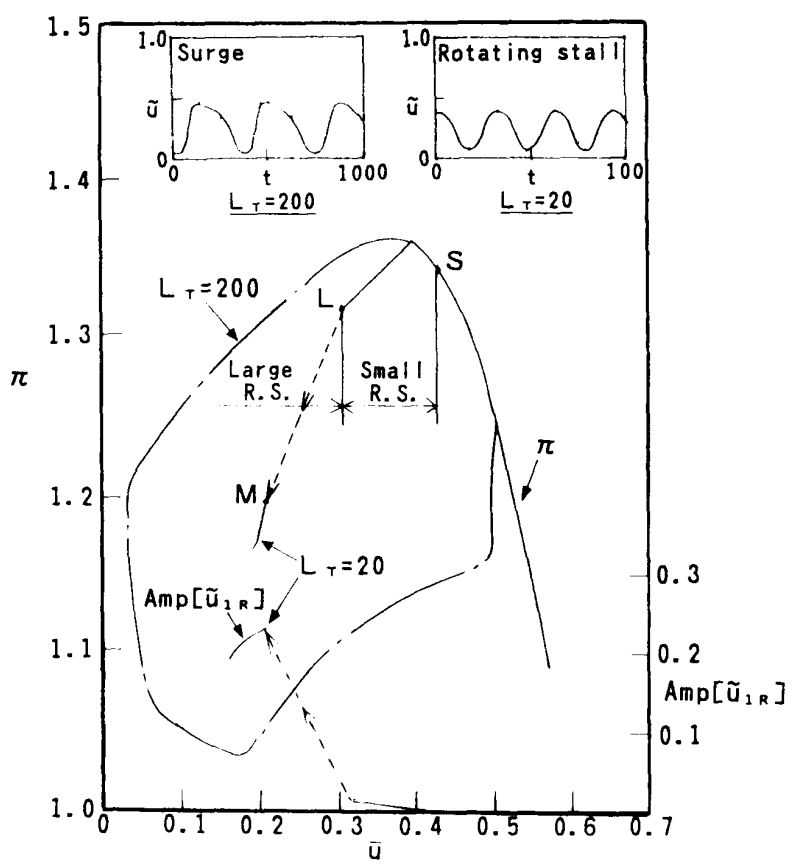

Fig. 5 Effects of throttle duct length on post - stall transients

the left side of point $\mathrm{S}$. These results qualitatively agree with those obtained using the fundamental model which represents the compressibility in terms of B-parameter; that is, surge tends to occur when the $\mathrm{B}$-parameter is large and rotating stall tends to occur when the B-parameter is small. The amplitude of the disturbance velocity at the first rotor blade, $A_{\mathrm{mp}}[u]$, is also shown as a function of the change in $u$ in the lower part of the figure. $A_{\mathrm{mp}}[u]$ represents the magnitude of the rotating stall. Rotating stall with small disturbance velocity occurs between points $\mathrm{S}$ and L. As $u$ decreases, $A_{\mathrm{mp}}[u]$ increases. When the operating point reaches point $\mathrm{L}$, it suddenly shifts to point $\mathrm{M}$, as shown by the dotted line. A sudden drop in $\pi$ occurs with constant throttle coefficient, and the disturbance velocity increases rapidly. The time history of the local flow velocity becomes a sine wave. Two types of rotating stall are obtained according to the amplitude of the disturbance velocity. In this study, we call rotating stall with small disturbance velocity "small R.S." (rotating stall) and rotating stall with large disturbance velocity "large R.S.". The different modes of rotating stall have been investigated (Ref. (4)), and it is often said that the interference of blade rows can induce two modes of rotating stall.

It has thus been found that the length of the throttle duct is one of the factors influencing the mode of unstable behavior and that there exist two types of rotating stall which differ in magnitude. It has also been verified that the present model can be used to simulate not only surge and rotating stall but also 
large and small rotating stalls.

\section{2 Total pressure loss and deviation angle effect}

Figure 6 shows how the steady and unsteady compressor characteristics $\pi-u$ differ with changes in the shapes of the total pressure loss and deviation angle characteristics, which are represented by $\beta_{2}$. The shapes influence not only the steady performance but also the unsteady performance. It is reasonable that the maximum pressure ratio increases as $\beta_{2}$ increases. The inception points of rotating stall change in correspondence to the points of the maximum pressure ratio. It should be noted that a small $\mathrm{R}$. $\mathrm{S}$. occurs on the negative slope of the compressor performance curve when $\beta_{2}=30.0$ and 37.5 , whereas it occurs near the maximum point when $\beta_{2}=45.0$. The shapes of total pressure loss and deviation angle characteristics have a strong influence on the inception position on the performance curve as well as the performance curve itself. This result supports the fact that stalling rarely occurs when the maximum pressure ratio is high. Figure 6 also indicates that the points $M_{1}, M_{2}$, and $M_{3}$, where large R.S. starts to occur shift with changes in $\beta_{2}$.

Figure 7 shows how the inception margin $M$ and amplitude of disturbance velocity $u$ due to large R.S. change with changes in $\beta_{2}$. The inception margin is calculated using the equation shown in Fig. 7, assuming that the pressure ratio is the design value $\pi_{D}=1.3$. As $\beta_{2}$ increases, both $M$ and $u$ increase. This indicates that a deterioration in compressor performance can

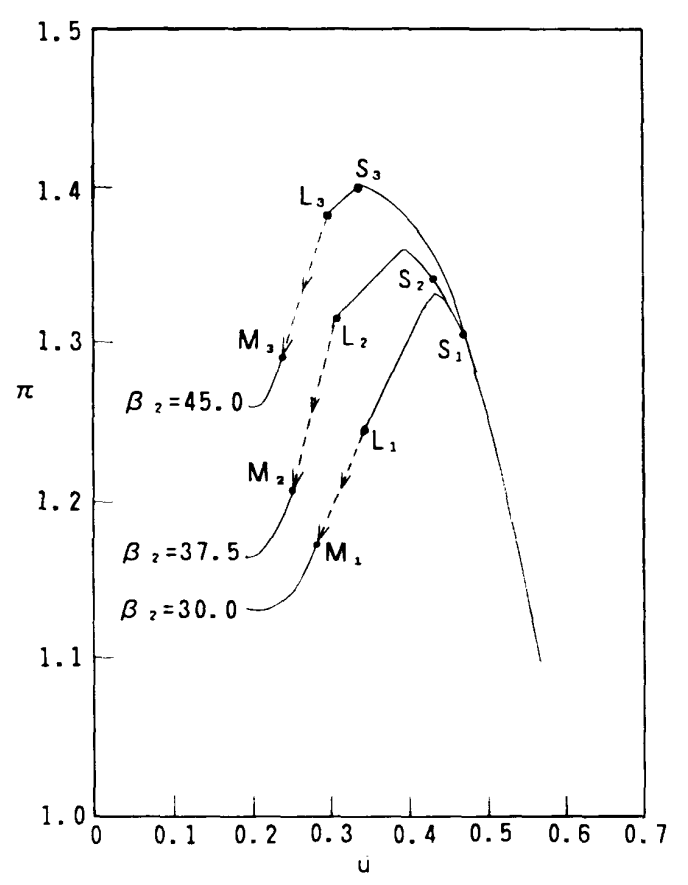

Fig. 6 Effects of total pressure loss and deviation angle on inception point lower the inception margin. The change in $u$ appears to be related to the pressure ratio at the operating points where large R.S. occurs (i.e., point $\mathrm{M}$ in Fig. 6) although further investigation is necessary to confirm this.

It has thus been found that a deterioration in the compressor performance lowers the inception margin of unstable behavior.

\section{3 IGV outlet flow angle effect}

Figure 8 shows how the steady and unsteady compressor characteristics $\pi-u$ change with changes in the IGV outlet flow angle $\gamma_{I C v}$, which changes the mass flow in the compressor. It is reasonable that the maximum pressure ratio and flow velocity in the unstalled region increase as $\gamma_{I G V}$ decreases. The inception points of rotating stall change in correspondence to the points of maximum pressure ratio, but the inception flow velocity is almost constant regardless of $\gamma_{I G V}$. In other words, the change in mass flow has little influence on the difference between the flow velocity at the maximum pressure ratio and the inception flow velocity. Figure 8 also shows that the pressure ratios at the points where large R. S. occurs, represented by $M_{1}, M_{2}$, and $M_{3}$ are almost the same regardless of $\gamma_{I G V}$.

Figure 9 shows how the inception margin $M$ and amplitude of disturbance velocity $u$ change with changes in $\gamma_{I c v}$. The inception margin is calculated in the same way as in the total pressure loss case, assuming that the design pressure ratio $\pi_{D}=1.3$ and

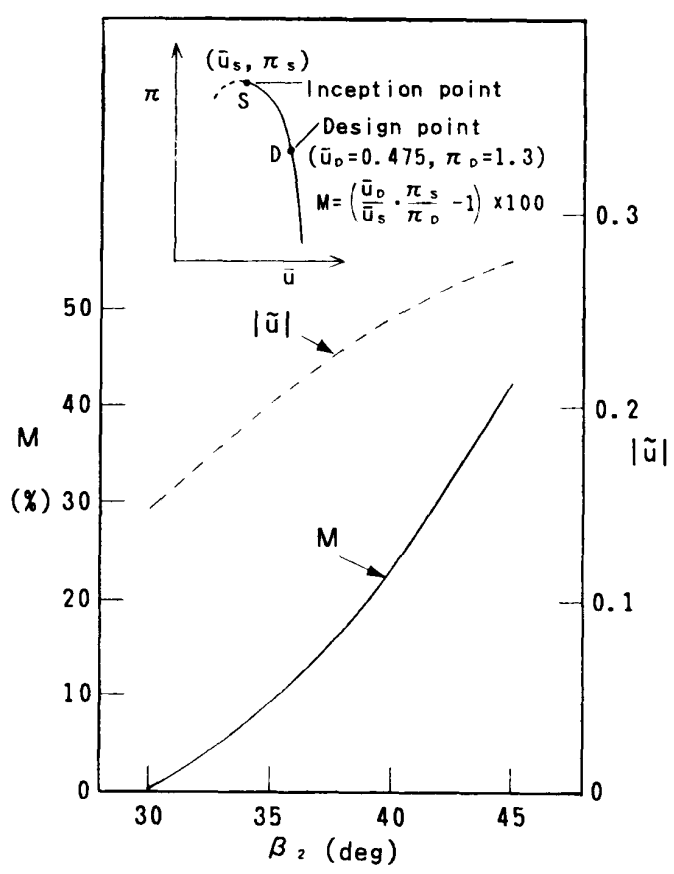

Fig. 7 Effects of total pressure loss and deviation characteristics on inception margin and disturbance velocity 
using the flow velocity corresponding to $\pi_{D}=1.3$ in each case. As $\gamma_{I G V}$ increases, $M$ decreases. This is because the design flow velocity decreases with increasing $\gamma_{I G}$, indicating that a larger mass flow results in a greater margin. On the other hand, $u$ is almost constant regardless of $\gamma_{I G V}$. This also supports the idea that the change in $u$ is related to the pressure ratio at the operating points where large $\mathrm{R}$. S. occurs,

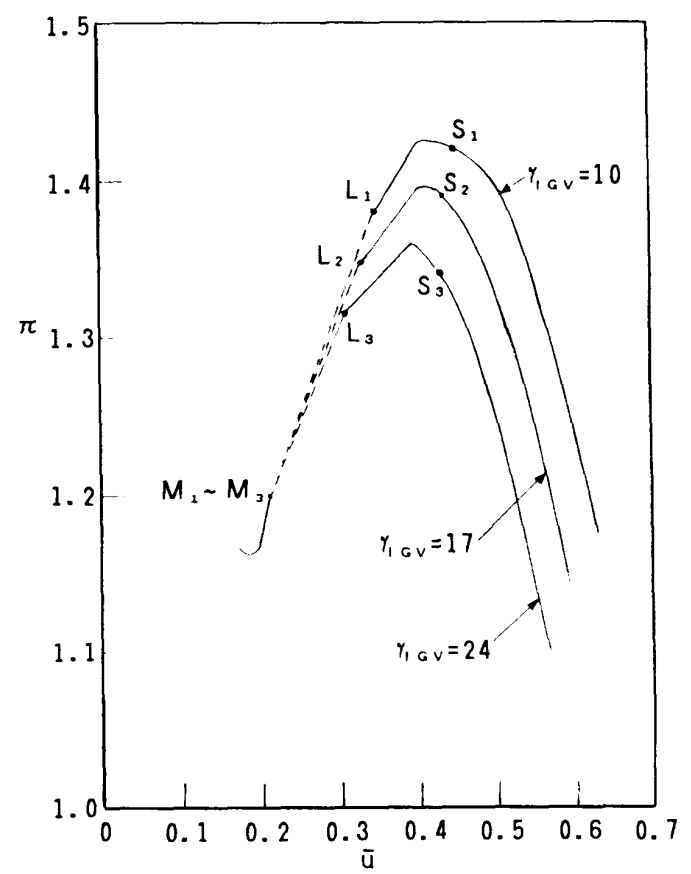

Fig. 8 Effects of IGV outlet flow angle on inception point

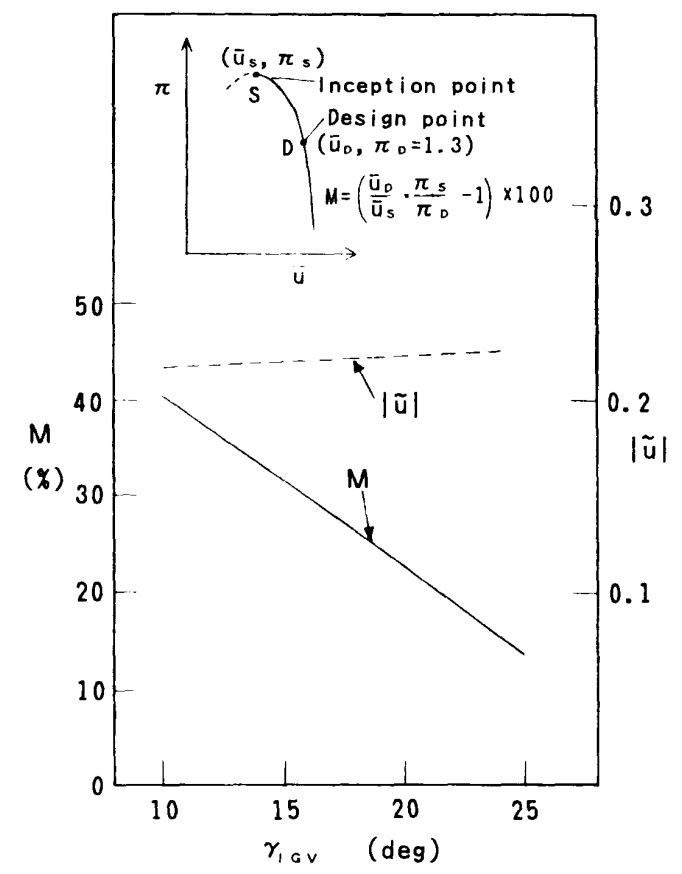

Fig. 9 Effects of IGV outlet flow angle on the inception margin and the amplitude of disturbance velocity as we suggested in subsection 4.2 when discussing the $\beta_{2}$ effect.

It has thus been found that the larger mass flow obtained at smaller IGV outlet flow angles results in a bigger inception margin of unstable behavior and that the IGV outlet flow angle has little influence on the magnitude of rotating stall.

\section{4 Inlet flow distortion effect}

Figure 10 shows how the inception margin $M$ and amplitude of disturbance velocity $u$ change with changes in circumferential inlet distortion of axial flow velocity $\varepsilon$. The inception margin is calculated in the same way as in subsections 4.2 and 4.3. $M$ decreases as $\varepsilon$ increases, indicating that inlet distortion lowers the inception margin, however the inlet distortion has less influence on the inception margin than the deterioration in compressor performance. When $\varepsilon$ is small, $u$ is almost constant regardless of changes in $\varepsilon$, but for $\varepsilon>0.05, u$ increases gradually as $\varepsilon$ increases. It is assumed that the amplitude of inlet distortion becomes comparable to that of disturbance velocity due to rotating stall when $\varepsilon$ is greater than 0.05 .

It has thus been found that inlet distortion lowers the inception margin of unstable behavior.

\section{5 Bleeding effect}

Figure 11 shows how the inception margin $M$ and amplitude of disturbance velocity $u$ change with changes in bleeding $Q_{B}$, which is normalized with respect to the main flow $Q$. This relation was calculated for a 2-stage compressor in which the blade

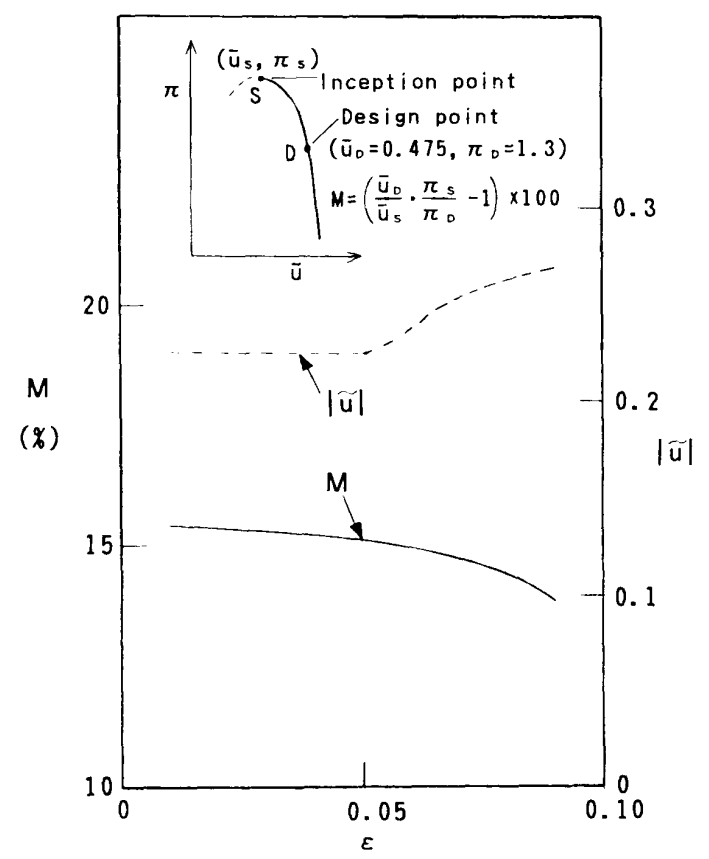

Fig. 10 Effects of inlet distortion on inception margin and disturbance velocity 


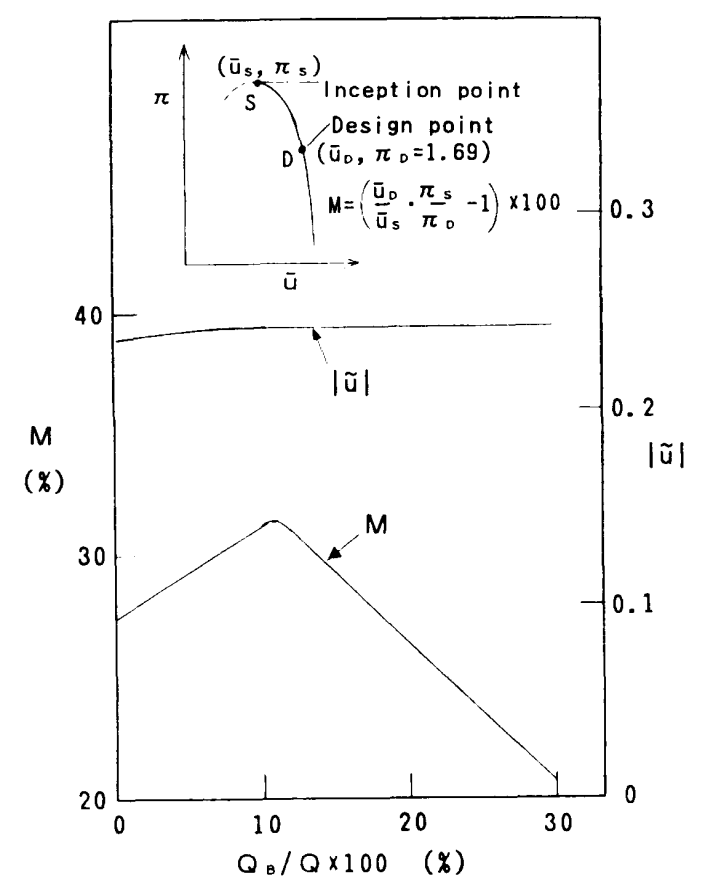

Fig. 11 Effects of bleeding on inception margin and disturbance velocity

angles of the second rotor and stator blades are 5 degrees greater than the design angles in order to produce a choke state. This state is often observed in multistage axial compressors when the rotational speed is less than the design speed, where rotating stall occurs in the front stages because of a choke state in the back stages. Bleeding is one of the most effective ways to reduce the range of speed within which rotating stall occurs. The inception margin is calculated in the same way as in the preceding subsections. This figure shows that there is an optimal $Q_{B}$, at which $M$ is the highest in the selected range of bleeding. In other words, the inception margin does not necessarily increase as bleeding increases. It is necessary to choose the appropriate amount of bleeding, and $u$ is almost constant regardless of $Q_{B}$.

It has thus been found that there is an optimal bleeding with regard to the inception margin of unstable behavior.

\section{6 Comparison with test results}

The first comparison was made for a single-stage low-speed compressor consisting of an entrance duct, blade rows, a throttle duct, and a throttle. The blade rows begin with IGVs and end with EGVs. An NACA 65 -series profile is used for both the rotor and the stator blades. The correlation of the total pressure loss coefficient and the deviation against the incidence were determined from the performance test results of the blade row in the unstalled situation. They were modified using the correlation of Takata et

\section{Test result}

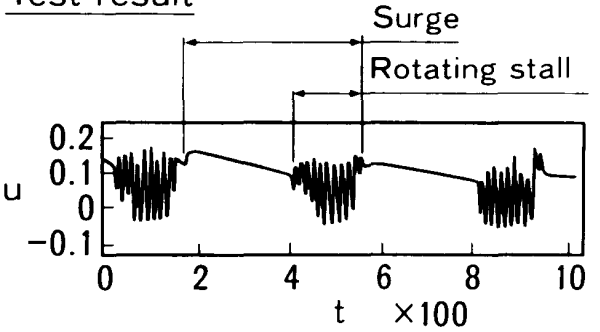

Numerical result

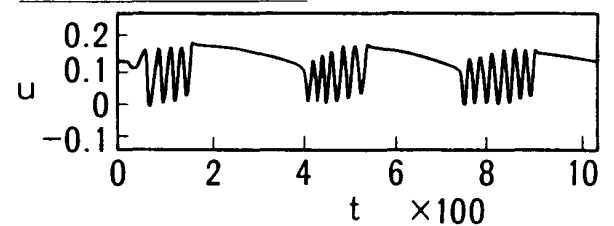

\begin{tabular}{|l|c|c|c|}
\hline & $\begin{array}{c}\text { Surge } \\
\text { period }\end{array}$ & $\begin{array}{c}\text { Propagation } \\
\text { speed }\end{array}$ & $\begin{array}{c}\text { Amplitude } \\
\text { of } u\end{array}$ \\
\hline Test result & 384 & 0.45 & 0.23 \\
Numerical result & 354 & 0.25 & 0.20 \\
\hline
\end{tabular}

Fig. 12 Calculated and test results at surge in low speed single-stage compressor

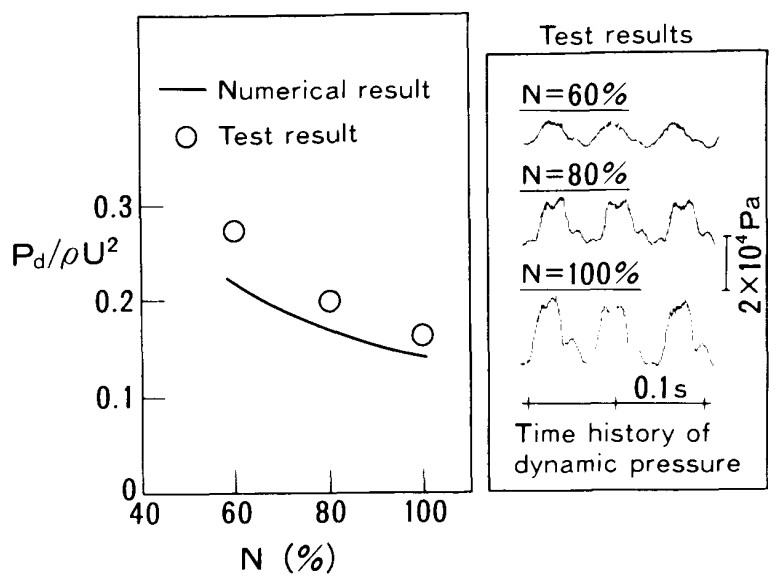

Fig. 13 Comparison of dynamic pressure amplitude dur ing surge in high speed single- stage compressor

al. ${ }^{(9)}$ for the stalled situation. Because the characteristics in a stalled situation are unknown, a cubic function was assumed for the total pressure loss and a parabolic function was assumed for the deviation angle.

Surge and rotating stall were observed simultaneously during a surge test at $80 \%$ design speed, and calculations were performed under the same conditions. Figure 13 shows the calculated and measured (with a hot-wire anemometer, located at the mean radii in front of the IGV) time histories of local flow velocity. Figure 12 shows calculated and measured surge period, propagation speed of rotating stall, and the amplitude of disturbance velocity. The waveforms determined using the proposed model indicate 
simultaneous occurrence of surge and rotating stall, and the calculated amplitude of flow velocity agrees well with the test results. The surge periods are almost the same in both cases, but the propagation speeds are different. The boundary conditions for the disturbance velocity are thought to be the source of this difference.

A comparison is also conducted for a single-stage high-speed compressor. The configurations of the ducts and plenum are the same as those of the previous low-speed compressor. This compressor is highly loaded compared with the previous one, by using a DCA (double circular arc) profile for the rotor blade. Surge tests were conducted at $60 \%, 80 \%$, and $100 \%$ speed. In this compressor, only surge occurred at all of these speeds as shown in Fig. 13. In the calculations, the correlation of the total pressure loss coefficient and the deviation angle against the incidence angle were selected to be almost the same as those for the previous compressor except for some improvements on unstalled performance, which have negligible effects on the post-stall behavior. Figure 13 also shows a comparison of the dynamic pressure amplitudes between the test and numerical results during surge at various speeds. The dynamic pressure is normalized by $\rho U_{2}$. The numerical results tend to give lower amplitudes than the test results. The difference increases with decreasing speed. However, the difference between the two sets of results is within

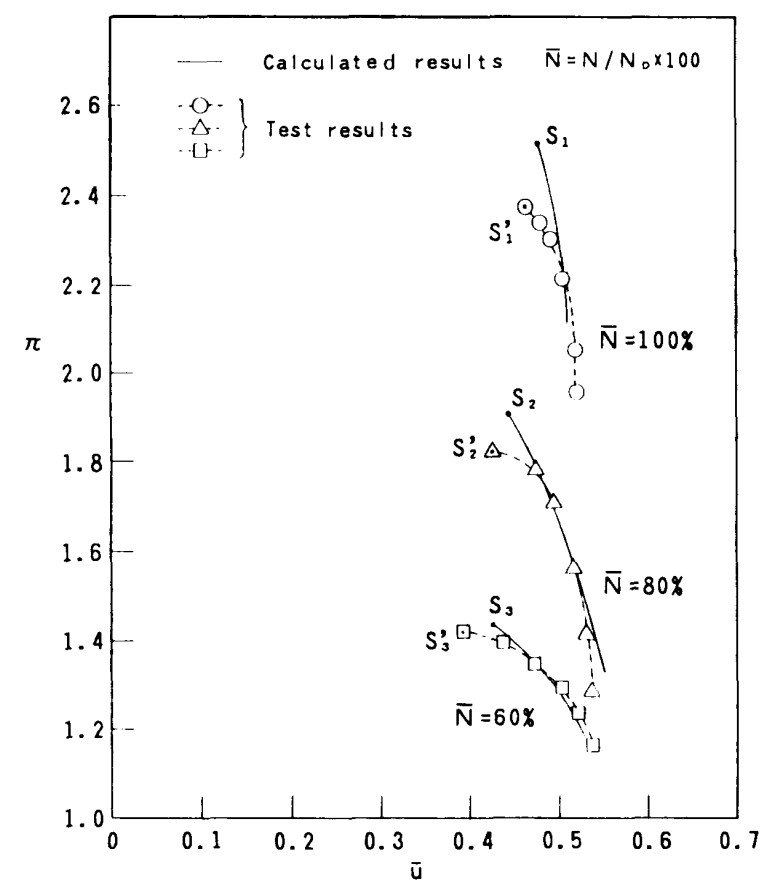

Fig. 14 Calculated and test results for steady performance and inception points in 3 stage compressor
$20 \%$, which is considered to be acceptable for the prediction of blade vibration.

Figure 14 shows the test and calculated results for steady performance and the inception points at $60 \%$, $80 \%$, and $100 \%$ design speeds in the 3 -stage compressor. In the regions of low pressure ratio, the calculated results agree with the test results. As the pressure ratio increases, the differences tend to increase at all speeds. The inception points obtained by calculation differ by $2-6 \%$ from those obtained from the tests. These errors are mainly due to the difference in the total pressure loss and deviation angle characteristics. In practice, it is quite difficult to estimate the characteristics of real compressors, the performance of which may change with time due to fouling on the blade surface. Further investigations are therefore necessary.

It has thus been verified that the present model is applicable to predict the unstable behavior of multistage axial compressors and that it can be used to quantitatively predict the amplitude and inception points of unstable behavior.

\section{Conclusions}

The effects of various parameters on the inception and post-stall characteristics of surge and rotating stall in multistage axial compressors have been investigated using a proposed numerical model, and the following results have been obtained.

(1) The length of the throttle duct is one of the factors influencing the mode of unstable behavior.

(2) There are two types of rotating stall that differ in magnitude.

(3) A deterioration in compressor performance lowers the inception margin of unstable behavior.

(4) Larger mass flow resulting from smaller IGV outlet flow angles results in a larger inception margin of unstable behavior. IGV outlet flow angle has little influence on the magnitude of rotating stall.

(5) Inlet distortion lowers the inception margin of unstable behavior.

(6) Bleeding can be optimized to make the inception margin of unstable behavior optimum.

The following points have been verified.

(1) The present model can be used to simulate not only surge and rotating stall but also large and small rotating stalls.

(2) The present method is applicable to multistage axial compressors and can be used to quantitatively predict the inception points of unstable behavior.

The usefulness of the present model has been verified. 


\section{Acknowledgment}

We thank Professor Franklin K. Moore of Cornell University for his informative suggestions in the first stage of this research.

\section{References}

(1) Hagiwara, N., Yoneyama, M., Ishii, H., Matsuura, Y. and Kashiwabara, Y., An Analysis of Blade Vibration of an Axial Flow Compressor during Rotating Stall, Turbomachinery, Vol. 13, No. 4 (1985), p. 193.

(2) Matsuura, Y., Katoh, Y., Kashiwabara, Y., Hagiwara, N., Ishii, H. and Tokunaga, K., An Experimental Study of Unsteady Flow in Multistage Axial-Flow Compressors, Proc. of CIMAC '87, T10 (1987), p. 1.

( 3 ) for example, Greitzer, E. M., Review-Axial Compressor Stall Phenomena, ASME Jour. Fluids Eng., Vol. 102 (1980), p. 134.

(4) Moore, F. K. and Greitzer, E. M., A Theory of Post-Stall Transients in Axial Compression Sys- tems: Part 1, Development of Equations, ASME 85-GT-171 (1985), p. 1.

(5) Greitzer, E. M. and Moore, F. K., A Theory of Post-Stall Transients in Axial Compression Systems: Part 2, Application, ASME 85-GT 172 (1985), p. 1.

(6) Moore, F. K., Stall Transients of Axial Compres sion Systems with Inlet Distortion. AIAA-85 1348 (1985), p. 1.

( 7 ) Ishii, H. and Kashiwabara, Y.. A Study on Surge and Rotating Stall in Axial Compressors (A Sum. mary of the Measurement and Fundamental Analysis Method), JSME Int. J., Ser. II, Vol. 32, No. 4 (1989), p. 583

(8) Ishii, H. and Kashiwabara, Y., Study on Surge and Rotating Stall in Axial Compressors (3rd Report. Numerical Model for Multiblade-Row Compressors), Trans. Jpn. Soc. Mech. Eng., (in Japanese), Vol. 59, No. 560, B (1993), p. 1127.

(9) Takata, H. and Nagano, S., Nonlinear Analysis of Rotating Stall, ASME Jour. of Eng. for Power, Vol. 94, No. 4 (1972), p. 279. 